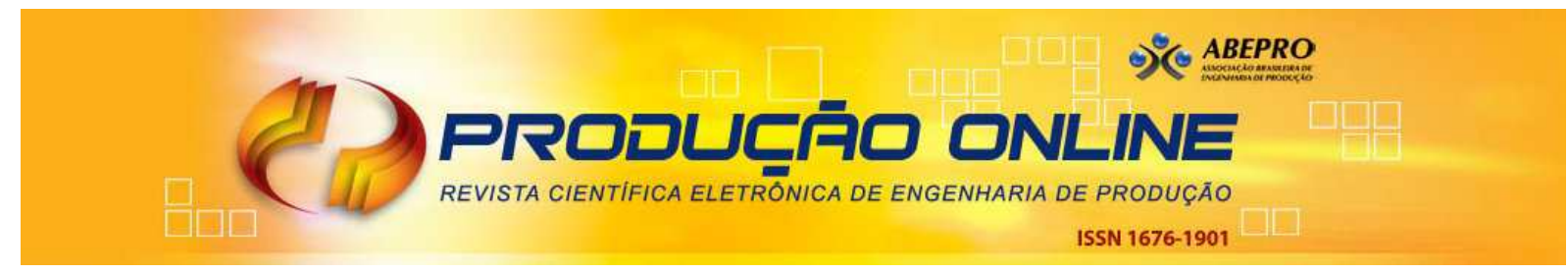

\title{
AVALIAÇÃO DA IMPLANTAÇÃO DA MANUFATURA ENXUTA: UMA ANÁLISE DOS ANAIS DO ENEGEP
}

\section{EVALUATION OF LEAN MANUFACTURING IMPLEMENTATION: REVIEW OF THE PROCEEDINGS OF THE ENEGEP}

\author{
Olga Maria Formigoni Carvalho Walter* olgaformigoni@gmail.com \\ Dalvio Ferrari Tubino*tubino@deps.ufsc.br \\ *Universidade Federal de Santa Catarina - UFSC, Florianópolis, SC
}

\begin{abstract}
Resumo: Por meio de uma revisão bibliográfica dos Anais do Encontro Nacional de Engenharia de Produção (ENEGEP), nos últimos 10 anos de realização do evento, este artigo tem como objetivo identificar o perfil da pesquisa brasileira com relação a métodos que avaliam a implantação da Manufatura Enxuta (ME), propondo uma classificação para os métodos analisados. Foram identificados 28 artigos que tratam especificamente da avaliação da implantação da ME, o que corresponde a 16 métodos de avaliação, pois alguns destes foram encontrados em mais de um artigo. Os resultados mostram que o tipo de pesquisa mais utilizado é o estudo de caso. As práticas enxutas mais abordadas nos métodos são: Ferramentas da Qualidade, Equipes Multifuncionais, Troca Rápida de Ferramentas, Melhoria Contínua e Gestão Visual. A presença do pesquisador foi constatada em mais da metade dos estudos analisados e menos da metade apresentou 0 instrumento de coleta de dados completo. Além disso, a maior parte dos artigos avalia a ME na indústria, especificamente no chão de fábrica. Este artigo será útil tanto para pesquisadores quanto para profissionais da área de Engenharia de Produção interessados em avaliar o progresso da implantação da ME, pois apresenta o perfil de todos os métodos apresentados no ENEGEP, o que reflete 0 andamento da pesquisa brasileira sobre $o$ assunto.
\end{abstract}

Palavras-chave: Manufatura Enxuta. Método de Avaliação. Práticas Enxutas. Implantação.

Abstract: Through a review of the proceedings of the National Meeting of Production Engineering (ENEGEP) over the past 10 years, this article aims to identify the profile of Brazilian research regarding methods that assess the implementation of Lean Manufacturing (LM), proposing a classification for the methods analyzed. We identified 28 articles that specifically address the assessment of the implementation of LM, which accounts for 16 evaluation methods because some methods have been found in more than one article. The results show that the type of research most widely used is the case study. Lean practices more addressed are: Quality Tools, Multifunctional Teams, Quick-Changeover Techniques, Continuous Improvement and Visual Control. The presence of the researcher was found more than half of the studies analyzed. Less than half showed the full data collection instrument. Moreover most of the papers evaluate the LM industry, specifically on the shop floor. This article will be useful both to researchers and to professionals in Production Engineering interested in evaluating the progress of LM implementation, because it shows the profile of all the methods presented in ENEGEP, which reflects the progress in the Brazilian research on the subject.

Key-words: Lean Manufacturing. Evaluation Method. Lean Practices. Implantation. 


\section{INTRODUÇÃO}

Desde que a Manufatura Enxuta (ME) se difundiu pelas indústrias, são vários os estudos que procuram descobrir a melhor maneira de sua implantação (AHLSTRÖM, 1998; MORIONES; PINTADO; CERIO, 2008). Porém, na última década, principalmente no Brasil, percebe-se uma crescente preocupação em avaliar os impactos da ME após sua implantação (DURAN; BATOCCHIO, 2003; CARDOZA; CARPINETTI, 2005; LUCATO; MAESTRELLI; VIEIRA JÚNIOR, 2006; SAURIN; FERREIRA, 2008, 2009).

$\mathrm{Na}$ literatura internacional, diversos estudos como Karlsson e Ahlström (1996), Sánchez e Pérez (2001) e Soriano-Meier e Forrester (2002) têm sido desenvolvidos no sentido de auxiliar as empresas a avaliarem o progresso de seus esforços em direção à ME.

Karlsson e Ahlström (1996) estabelecem um conjunto de indicadores (determinantes e medidas) que permitem avaliar a extensão pela qual os princípios da ME são adotados, o que reflete as mudanças ocorridas com a implantação da ME. A estrutura básica do método de avaliação de Sánchez e Pérez (2001) é uma lista de verificação que segue os princípios da ME, composta por um conjunto de seis princípios desdobrados em trinta e seis indicadores que contribuem para a melhoria do desempenho das empresas. O método desenvolvido por Soriano-Meier e Forrester (2002) tem como objetivo examinar a relação entre a adoção dos princípios enxutos nas empresas e o comprometimento da gerência com a ME.

É relevante a necessidade de um sistema que avalie a ME para identificar pontos fracos de sua implantação que requerem maior acompanhamento. Nesse sentido, este artigo tem como objetivo identificar no contexto da pesquisa brasileira, os métodos científicos que avaliam a ME e explorar suas principais características. Para isso, utiliza-se, como fonte de dados, os artigos publicados nos Anais do ENEGEP. Cabe ressaltar que não foram considerados artigos que avaliavam a implantação de uma prática isolada da ME, tal como a utilização do Kanban ou 5S.

A escolha pelos Anais do ENEGEP como fonte de consulta de publicações ocorreu principalmente por ser considerado o evento de Engenharia de Produção mais importante no país, no qual são discutidos e relatados contribuições de pesquisas importantes relacionadas à área, incluindo, principalmente, pesquisas de 
nível de especialização e pós-graduação. Outro motivo é o fato de que seu alcance, abrangência e relevância refletem o estado da arte da pesquisa brasileira na área (BERTO; NAKANO, 2000).

Assim, realizou-se uma pesquisa bibliográfica e propôs-se uma classificação, a partir das características das publicações identificadas. Foram considerados seis parâmetros: práticas da ME (que são enfatizadas no método de avaliação), tipo de metodologia científica utilizada, método aplicado à ME implantada, presença do pesquisador na aplicação do método, apresentação do instrumento de coleta de dados e, finalmente, se o método apresenta alguma classificação de quão enxuta se apresenta a empresa avaliada.

É importante destacar que este artigo preocupa-se com a estruturação dos métodos de avaliação da ME, não focando nos resultados apresentados, visto que o resultado da implantação da ME varia de acordo com as particularidades da empresa na qual foi aplicado.

Este trabalho será útil tanto para pesquisadores quanto para profissionais da área de Engenharia de Produção interessados em avaliar o progresso da implantação da ME, pois apresenta o perfil de todos os métodos apresentados no ENEGEP, o que reflete $o$ andamento da pesquisa brasileira sobre $o$ assunto.

Este artigo está estruturado da seguinte forma: partindo-se da introdução e objetivos aqui apresentados, segue-se para a seção 2 em que são detalhados os procedimentos metodológicos adotados. A forma de classificação utilizada para extrair as principais características dos artigos analisados é detalhada na seção 3 . $\mathrm{Na}$ quarta seção, os métodos de avaliação da ME identificados nos Anais do ENEGEP são brevemente descritos. Na quinta seção procede-se a análise dos artigos identificados, mediante classificação proposta na seção 3. Finalmente, na seção 6, são expostas as conclusões e considerações finais.

\section{METODOLOGIA}

Este trabalho é caracterizado como teórico-conceitual e realiza uma revisão bibliográfica sobre estudos que avaliam a implantação da Manufatura Enxuta (ME) mais recentemente discutidos na literatura brasileira. É um estudo exploratório, que 
se constitui em fonte secundária, na qual a coleta de informações é realizada por meio de documentação (MEDEIROS, 2007; GIL, 2010).

O levantamento de dados foi realizado pela consulta nos Anais do ENEGEP e também contou com o apoio de consulta eletrônica aos artigos publicados no site da Associação Brasileira de Engenharia de Produção (ABEPRO). O período considerado na análise da revisão bibliográfica compreende os últimos dez anos de realização do evento - de 2001 a 2010.

A condução desta pesquisa ocorreu da seguinte forma: paralelamente à pesquisa dos Anais do ENEGEP, através do site da ABEPRO, foram consideradas as seguintes palavras-chave, individuais e combinadas, para a busca de artigos que avaliam a ME: lean, enxuta, manufatura enxuta, produção enxuta, lean manufacturing, avaliação, implantação, método, implementação, práticas enxutas, avaliação, indicadores, modelo de avaliação e ferramentas da produção enxuta. Esta pesquisa por palavras-chave retornou, em média, 700 artigos por ano.

A partir dessa coleta, foram selecionados, de cada ano, somente artigos diretamente relacionados à ME, resultando em um total de 490 publicações. Ainda, nessa fase, foi necessária mais uma análise, com o intuito de excluir os artigos duplicados. Posteriormente, foram analisados os resumos desses artigos, com o objetivo de identificar publicações que avaliassem a implantação da ME com maior abrangência, ou seja, foram excluídos da análise artigos que avaliavam uma ferramenta específica da ME, como por exemplo, avaliação do uso do Kanban e artigos que detalhavam as melhorias obtidas com a implantação de práticas isoladas, tais como Troca Rápida de Ferramenta e 5S. Assim, foram obtidos, para o período de 2001 a 2010, 28 artigos diretamente relacionados à avaliação da implantação da ME.

A quantidade de artigos da revisão publicados, de acordo com o ano, é mostrada na Figura 1. Verifica-se que não houve nenhuma publicação no primeiro ano investigado (2001) e observa-se que houve a oscilação no crescimento de publicações ao longo do período com maior concentração nos últimos três anos (2008, 2009 e 2010). 
Figura 1 - Quantidade de publicações distribuídas ao longo do período analisado

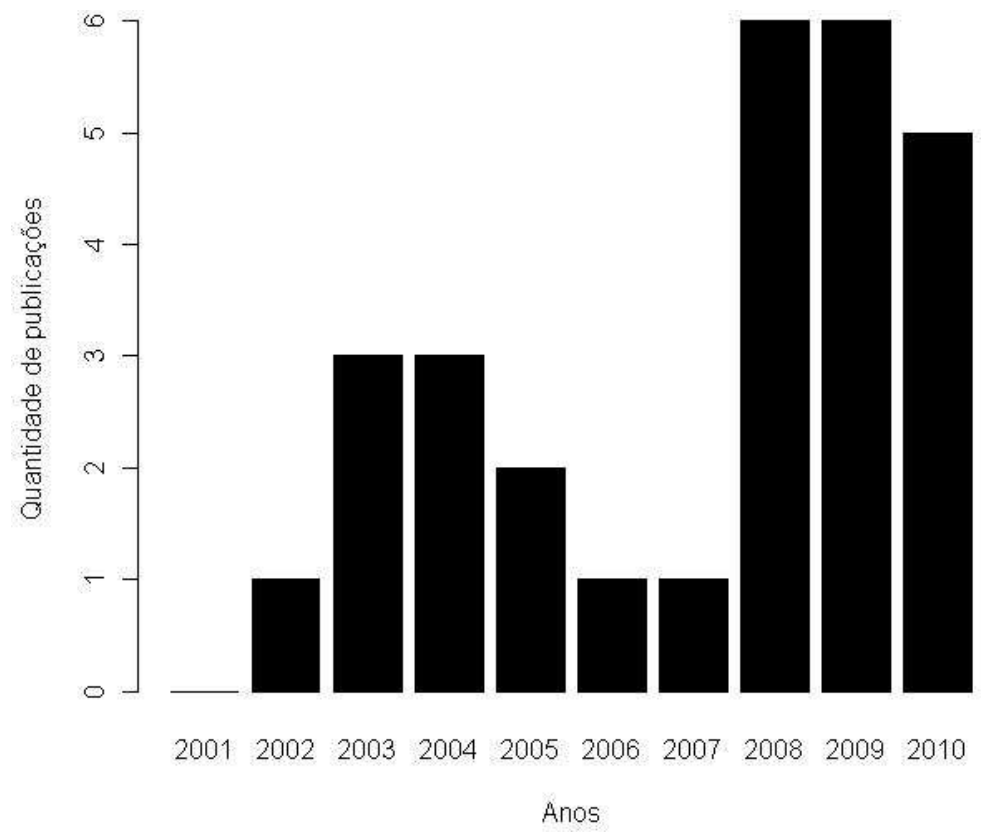

Uma hipótese, ainda sem comprovação, para ausência de publicações no início do período analisado, que se pode levantar é o fato de que, nessa época, no Brasil, tanto as pesquisas como as empresas ainda estavam em fase inicial de implantação da $\mathrm{ME}$, sem resultados relevantes para o desenvolvimento e publicação de métodos de avaliação.

\section{CLASSIFICAÇÃO DAS PUBLICAÇÕES IDENTIFICADAS}

Nesta seção é apresentado o sistema de classificação proposto para a literatura coletada. Esta classificação faz-se necessária, pois a partir dela é possível identificar as principais características e o perfil dos trabalhos publicados.

\subsection{Sistema de Classificação Proposto}

O sistema de classificação é composto por seis características gerais, identificadas nas publicações pesquisadas:

a) práticas da ME enfatizadas no método de avaliação;

b) abordagem metodológica empregada; 
c) ME implantada ou não;

d) se há a presença do pesquisador na aplicação do método;

e) se apresenta o instrumento de coleta de dados;

f) se apresenta alguma classificação ou grau de escala de implantação da ME.

Para a categoria que se refere às práticas enxutas enfatizadas no trabalho, foram consideradas importantes referências de ME como Womack e Jones (1992), Womack, Jones e Roos (1992), Takahashi e Osada (1993), Imai (1994), Shingo (1996, 2000), Ohno (1997), Rother e Harris (2002) e Liker (2005) das quais foram extraídos os princípios mais importantes. Pelo fato de os artigos revisados apresentarem várias terminologias para as práticas enxutas utilizadas nos trabalhos, optou-se por considerar uma ampla gama de conceitos propostos pelos autores, como práticas enxutas, indicadores enxutos, princípios, metodologias e ferramentas e condensá-los, quando possível, em um único indicador para evitar o uso de diferentes nomenclaturas para práticas que possuem o mesmo significado. Por exemplo, Heijunka foi incorporado à prática "Nivelamento da Produção" e instrução de trabalho foi incorporado à prática "Operações Padronizadas".

A abordagem metodológica empregada diz respeito à caracterização do tipo de metodologia científica utilizada nas bibliografias analisadas neste estudo. As abordagens metodológicas são baseadas nos trabalhos de Filippini (1997), Berto e Nakano (2000) e Miguel (2007), os quais apontam estudos de caráter teóricoconceitual, experimentos, modelagem, simulação, survey, estudo de caso e pesquisa-ação, como abordagens metodológicas típicas da engenharia de produção e gestão de operações.

O item Manufatura Enxuta implantada ou não, se refere à condição da ME, abrangendo três situações:

1) método para empresas que já tenham implantado a $M E$ e precisam de uma avaliação de sua implantação;

2) método para empresas que ainda não tenham implantado a $M E$ e precisam de apoio na sua implantação e;

3) ambas as situações citadas anteriormente, ou seja, método que pode ser utilizado tanto por empresas que já tenham implantado a ME quanto por aquelas que desejam implantá-la. 
Para os três últimos itens: presença do pesquisador na aplicação do método, apresentação do instrumento de coleta de dados e apresentação de alguma classificação ou escala de implantação da ME, optou-se apenas por identificar sua presença ou ausência utilizando indicadores Sim e Não.

\subsection{Classificação das Publicações sobre Avaliação da Manufatura Enxuta}

A classificação, quanto às seis categorias apresentadas previamente, é mostrada nas Tabelas 1 e 2. Na Tabela 1 são apresentadas as 22 práticas da ME consideradas pelos métodos analisados classificadas em ordem alfabética.

Tabela 1 - Práticas da Manufatura Enxuta presente nos métodos identificados

\begin{tabular}{|c|c|c|c|c|c|c|c|c|c|c|c|c|c|c|c|c|}
\hline \multirow{2}{*}{$\begin{array}{c}\text { Práticas da Manufatura Enxuta } \\
\text { enfatizada pelo método }\end{array}$} & \multicolumn{16}{|c|}{ Métodos de Avaliação da Manufatura Enxuta } \\
\hline & 1 & 2 & 3 & 4 & 5 & 6 & 7 & 8 & 9 & 10 & 11 & 12 & 13 & 14 & 15 & 16 \\
\hline $5 S$ & & $*$ & & & $*$ & $*$ & $*$ & $*$ & & $*$ & & & & & $*$ & $*$ \\
\hline Agregação de Valor & $*$ & & & & $*$ & & $*$ & & $*$ & & $*$ & & & & $*$ & $*$ \\
\hline Andon & & $*$ & & & & & & & & & & $*$ & & & $*$ & $*$ \\
\hline Autonomação, Jidoka & & & & & $*$ & $*$ & & & & & $*$ & $*$ & & & $*$ & \\
\hline Equipes Multifuncionais & & $*$ & $*$ & & $*$ & $*$ & $*$ & $*$ & $*$ & $*$ & $*$ & $*$ & & $*$ & & $*$ \\
\hline Ferramentas da Qualidade & & $*$ & & $*$ & $*$ & $*$ & $*$ & $*$ & $*$ & $*$ & $*$ & $*$ & $*$ & & $*$ & \\
\hline Fluxo Contínuo & & & $*$ & & * & & $*$ & & & & & & & $*$ & & $*$ \\
\hline Gestão Visual, Comunicação & & $*$ & & & $*$ & $*$ & $*$ & $*$ & & & $*$ & $*$ & & & $*$ & $*$ \\
\hline Integração de Fornecedores & & & & & $*$ & $*$ & $*$ & & & & & $*$ & & & $*$ & \\
\hline Kanban, Sistema Puxado, JIT & & $*$ & $*$ & & $*$ & $*$ & & & & $*$ & & $*$ & & & $*$ & $*$ \\
\hline Layout de Fábrica & & $*$ & & & & & $*$ & & & $*$ & & & & & & \\
\hline Manutenção Produtiva Total & & & & $*$ & $*$ & $*$ & $*$ & & $*$ & $*$ & & $*$ & & & $*$ & \\
\hline Mapeamento do Fluxo de Valor & & & $*$ & & & $*$ & & & & & & $*$ & & & & \\
\hline Melhoria Contínua, Kaizen & * & & $*$ & & * & $*$ & $*$ & & & $*$ & $*$ & $*$ & & & $*$ & $*$ \\
\hline Nivelamento da Produção & & $*$ & $*$ & & & * & & & $*$ & * & * & $*$ & & & * & \\
\hline Operações Padronizadas & & $*$ & & & & $*$ & & $*$ & $*$ & & $*$ & $*$ & & & $*$ & $*$ \\
\hline Poka yoke & & $*$ & & & & * & & $*$ & & & & $*$ & & & $*$ & \\
\hline Projeto/Desenv. de prod. flexível & & & & & & & $*$ & & $*$ & & & $*$ & & $*$ & $*$ & $*$ \\
\hline Segurança no Trabalho & $*$ & & & $*$ & & & & & & & & $*$ & $*$ & & & \\
\hline Satisfação no Trabalho & * & $*$ & & * & & & & $*$ & & & & $*$ & * & $*$ & & \\
\hline Tecnologia de Grupo & & & & & & $*$ & & & & & & $*$ & & & & \\
\hline Troca Rápida de Ferramentas & & * & & & $*$ & $*$ & $*$ & $*$ & $*$ & $*$ & $*$ & $*$ & & & $*$ & \\
\hline
\end{tabular}

(1) Giesta e Maçada (2002) e Giesta, Maçada e Lunardi (2003); (2) Pantaleão e Antunes Júnior (2003); (3) Sellitto, Borchard e Pereira (2003) e Sellitto et al. (2004); (4) Borges Júnior et al. (2004); (5) Dias, Fernandes e Godinho Filho (2004) e Fernandes, Godinho Filho e Dias (2005); (6) Ferreira e Saurin (2005), Nogueira e Saurin (2006) e Silva, Araújo e Gomes (2009); (7) Satolo e Calarge (2007) e Calarge, Satolo e Pereira (2009); (8) Batista, Muniz e Batista Júnior (2008); (9) Dal Forno et al. (2008), Tubino et al. (2008), Dal Forno et al. (2009), Silva, Elias e Tubino (2009) e Silva et al. (2010); (10) Francisco e Hatakeyama (2008, 2009); (11) Furini e Saurin (2008); (12) Nogueira e Casalinho (2008); (13) Cezar e Moita (2009); (14) Marodin e Saurin (2010); (15) Rosa et al. (2010a) e Rosa et al. (2010b); (16) Souza e Brandstetter (2010).

Através da Tabela 1 é possível identificar quais práticas da ME estão presentes em cada método. O método 12, que corresponde a publicação de 
Nogueira e Casalinho (2008), é o que aborda mais práticas; já o método 13, de Cezar e Moita (2009), abrange a menor quantidade de práticas.

A Tabela 2 apresenta as cinco categorias restantes, propostas de acordo com a seção 3.1, na qual os artigos estão dispostos em ordem cronológica e alfabética.

Tabela 2 - Característica dos artigos que avaliam a implantação da Manufatura Enxuta

\begin{tabular}{|c|c|c|c|c|c|}
\hline Publicação & $\begin{array}{l}\text { Metodo } \\
\text {-logia }\end{array}$ & $\begin{array}{l}\text { Implan- } \\
\text { tação } \\
\text { da ME }\end{array}$ & $\begin{array}{l}\text { Presen- } \\
\text { ça do } \\
\text { pesqui- } \\
\text { sador }\end{array}$ & $\begin{array}{c}\text { Instru- } \\
\text { mento } \\
\text { de } \\
\text { coleta }\end{array}$ & $\begin{array}{l}\text { Classificação } \\
\text { ou escala de } \\
\text { implantação }\end{array}$ \\
\hline Giesta e Maçada (2002) & EC & $\mathrm{I}$ & $\checkmark$ & $x$ & $x$ \\
\hline Giesta, Maçada e Lunardi (2003) & SU & I & $\checkmark$ & $\checkmark$ & $x$ \\
\hline Pantaleão e Antunes Júnior (2003) & * & i & ** & $\checkmark$ & $\checkmark$ \\
\hline Sellitto, Borchard e Pereira (2003) & EC & $\mathrm{NI}$ & * & $x$ & $x$ \\
\hline Borges Júnior et al. (2004) & * & 1 & * & $\checkmark$ & $x$ \\
\hline Dias, Fernandes e Godinho Filho (2004) & EC & INI & * & $\checkmark$ & $x$ \\
\hline Sellitto et al. (2004) & * & $\mathrm{NI}$ & * & $x$ & $x$ \\
\hline Fernandes, Godinho Filho e Dias (2005) & * & INI & * & $\checkmark$ & $x$ \\
\hline Ferreira e Saurin (2005) & EC & INI & $\checkmark$ & $x$ & $x$ \\
\hline Nogueira e Saurin (2006) & EC & 1 & $\checkmark$ & $x$ & $x$ \\
\hline Satolo e Calarge (2007) & SU & I & $x$ & $x$ & $\checkmark$ \\
\hline Batista, Muniz e Batista Júnior (2008) & EC & i & $\checkmark$ & $x$ & $x$ \\
\hline Dal Forno et al. (2008) & EC & INI & $\checkmark$ & $x$ & $\checkmark$ \\
\hline Francisco e Hatakeyama (2008) & * & INI & ** & $x$ & $x$ \\
\hline Furini e Saurin (2008) & EC & INI & ** & $\checkmark$ & $x$ \\
\hline Nogueira e Casalinho (2008) & TC & 1 & ** & $\checkmark$ & $x$ \\
\hline Tubino et al. (2008) & EC & INI & $\checkmark$ & $\checkmark$ & $\checkmark$ \\
\hline Calarge, Satolo e Pereira (2009) & SU & 1 & $x$ & $x$ & $\checkmark$ \\
\hline Cezar e Moita (2009) & * & 1 & * & $x$ & $x$ \\
\hline Dal Forno et al. (2009) & EC & INI & $\checkmark$ & $\checkmark$ & $\checkmark$ \\
\hline Francisco e Hatakeyama (2009) & * & INI & $\checkmark$ & $x$ & $x$ \\
\hline Silva, Araújo e Gomes (2009) & EC & INI & $\checkmark$ & $x$ & $x$ \\
\hline Silva, Elias e Tubino (2009) & EC & INI & $\checkmark$ & $\checkmark$ & $\checkmark$ \\
\hline Marodin e Saurin (2010) & EC & 1 & $\checkmark$ & $x$ & $x$ \\
\hline Rosa et al. (2010a) & SU & I & $x$ & $x$ & $\checkmark$ \\
\hline Rosa et al. (2010b) & SU & I & $x$ & $x$ & $\checkmark$ \\
\hline Silva et al. (2010) & EC & INI & $\checkmark$ & $\checkmark$ & $x$ \\
\hline Souza e Brandstetter (2010) & EC & INI & $\checkmark$ & $\checkmark$ & $x$ \\
\hline
\end{tabular}

NOTA: $(\checkmark)$ : Sim; (x): Não; (EC): Estudo de Caso; (SU) Survey; (TC): Teórico-conceitual;

(I): Manufatura Enxuta Implantada; (NI): Manufatura Enxuta Não Implantada; (INI): Manufatura Enxuta Implantada ou Não.

* Não foi possível identificar o aspecto analisado ou o artigo não especifica a informação considerada;

** Método não aplicado na prática.

Demais considerações sobre as características das publicações identificadas nas Tabelas 1 e 2 são detalhadas na seção 5 . 


\section{PUBLICAÇÕES IDENTIFICADAS SOBRE AVALIAÇÃO DA MANUFATURA ENXUTA}

A seguir, será feita uma breve descrição dos métodos pesquisados de acordo com a classificação apresentada na seção anterior, focando-se em duas categorias específicas da classificação proposta: Manufatura Enxuta implantada e se apresenta o instrumento de coleta de dados.

\subsection{Métodos para Empresas com Manufatura Enxuta já Implantada}

\subsubsection{Métodos que apresentam o instrumento de coleta de dados}

Giesta e Maçada (2002) propõem um método que tem como objetivo medir a satisfação dos funcionários inseridos no contexto da ME. $O$ instrumento é estruturado em cinco constructos: produtividade no trabalho, inovação no trabalho, satisfação no trabalho, controle gerencial e estima/auto-realização. Baseando-se nesses constructos, foi elaborado um questionário com 22 questões. Utilizou-se a escala Likert de cinco pontos, que foi aplicada, via estudo de caso, aos colaboradores de uma fábrica de tratores e retroescavadeiras.

Pantaleão e Antunes Júnior (2003) apresentam uma ferramenta de diagnóstico sobre a aprendizagem de uma organização a respeito dos princípios da ME. A estrutura do modelo se apóia em cinco fatores base: estratégia, estrutura, sistema de produção, indicadores e resultados. Cada fator base está relacionado a dez pontos chave (foco no cliente, liderança, organização, parcerias, arquitetura de informações, cultura de melhorias, função processo, função operação, indicadores de processos e operações e resultados do negócio), para o desenvolvimento da organização. Eles são avaliados por meio de uma série de questionários semiestruturados, de acordo com a seguinte classificação: "Produção em massa", "Sistema iniciante", "Sistema em desenvolvimento", "Sistema maduro" e "Excelência do sistema".

Borges Júnior et al. (2004) avaliam a implantação da ME por meio de cinco indicadores quantitativos (produtividade da mão-de-obra, qualidade: percentual de veículos sem defeito; segurança; absenteísmo e eficiência de manutenção). Esse 
método foi aplicado a uma linha específica de uma montadora de automóveis no Brasil.

Nogueira e Casalinho (2008) apresentam uma matriz com 87 indicadores distribuídos entre 15 práticas enxutas que abrangem tanto o nínvel operacional quanto o gerencial, indicando quando determinada prática deve aumentar e quando esta prática deve diminuir para alcançar a ME. O método não foi aplicado na prática.

\subsubsection{Métodos que não apresentam o instrumento de coleta de dados}

Giesta, Maçada e Lunardi (2003) avaliam o impacto da ME no trabalho com base na percepção dos funcionários, baseado no método de Giesta e Maçada (2002). O instrumento de coleta de dados é composto por um questionário de 18 questões, sendo operacionalizado por meio de escala tipo Likert de 5 pontos. Esse método foi aplicado via survey, em 4 empresas do setor metal mecânico, no Rio Grande do Sul.

Nogueira e Saurin (2006) utilizam o método desenvolvido por Ferreira e Saurin (2005) e o aplicam em uma empresa fornecedora de semi-eixos para montadoras de automóveis via estudo de caso. A condução do método foi estruturada sistematicamente da seguinte forma: entrevistas com gerentes envolvidos no processo de implementação; aplicação de um check-list de típicas práticas enxutas e elaboração de um ranking das práticas do check-list, em termos de grau de importância na percepção dos gerentes.

Satolo e Calarge (2007) propõem, por meio de survey, a determinação do grau de aderência à ME em seis empresas da região de Piracicaba/SP, baseandose na norma SAE J4000 (Identificação e mensuração de melhores práticas na implementação de uma operação enxuta). O método é estruturado em quatro etapas: 1) Compilação dos resultados, computando os dados para cada componente avaliado pela norma por empresa; 2) Associação das pontuações obtidas por cada empresa, segundo o peso atribuído pela norma; 3) Cálculo do porcentual representativo para cada elemento avaliado e; 4) Cálculo do grau de enxugamento das empresas. Para a determinação do grau de aderência à $M E$, é necessário realizar o somatório dos pontos relativos a três dos elementos que compõem a norma SAE J4000, por meio da Equação 1: 
(\%) Elemento $(x)=$ número de pontos atribuídos pela empresa $x$ 0,25 número máximo de pontos possíveis pelo elemento

Assim, o grau de aderência da empresa à ME pode ser calculado pelo somatório dos porcentuais obtidos para cada elemento, por meio da Equação 2:

$$
\text { GE empresa }=\Sigma \% \text { elemento }(1+2+3,4 \text { e } 5+6)
$$

Batista, Muniz e Batista Júnior (2008) utilizam o modelo de Gestão de Produção baseado no Conhecimento (MGP-C), que integra conceitos de gestão do conhecimento, organização da produção e organização do trabalho. O método é constituído por seis fatores (objetivo, estrutura, comunicação, treinamento, incentivo e característica pessoal) que potencializam a criação de condições favoráveis e de forma integrada. O objetivo é promover ações para melhores resultados à organização e às pessoas que nela trabalham. Este método foi aplicado em quatro empresas do grupo Toyota, a fim de avaliar como a ME é operada nas diferentes empresas selecionadas do grupo.

Marodin e Saurin (2010) propõem 15 aspectos associados aos princípios da ME. Tais aspectos são caracterizados como barreiras que impactam na implantação desse método. O método foi aplicado por meio de um estudo de caso em uma empresa que atua no ramo de componentes e sistemas de transmissão para veículos leves e pesados. Além de responder ao questionário semi-estruturado proposto pelo método, os entrevistados devem indicar se a lista das 15 barreiras propostas está presente na empresa, podendo também incluir novas barreiras na lista.

Por meio de um survey, Rosa et al. (2010a) propõem identificar em 38 empresas da região de Bauru-SP o nível de implementação das práticas de ME existente e se suas prioridades competitivas de produção (qualidade, rapidez, confiabilidade, flexibilidade e custo) influenciam na adoção destas práticas. Com o mesmo método e instrumento de coleta de dados, Rosa et al. (2010b) identificam o nível de adoção das práticas da ME nestas mesmas empresas. O instrumento de coleta de dados é composto por 26 questões que devem ser respondidas segundo 0 nível da aplicação das práticas e ferramentas da ME utilizadas, como: D desconheço; $\mathrm{NI}$ - não implantada; $\mathrm{Cl}$ - começando a implantar; IA - implantação avançada; I - implantada. O questionário aplicado foi ainda dividido em dois grandes 
grupos, G1 (eliminação de desperdícios e processos) e G2 (filosofia, melhoria contínua e aprendizado).

Calarge, Satolo e Pereira (2009), baseado em Satolo e Calarge (2007), apresentam os principais resultados e aspectos relativos a uma pesquisa survey conduzida com empresas do setor automobilístico do Brasil e Espanha, cujo objetivo consistia em determinar o grau de adequação destas empresas a ME.

Cezar e Moita (2009) baseiam-se em análise de cinco indicadores gerenciais do triênio, estabelecidos pela direção da empresa em que o método foi aplicado, para avaliar a implantação da ME. Os indicadores analisados são Qualidade (produtos rejeitados ou sucateados; produtos que passam pela inspeção final de sem sofrer retrabalho; reclamações à assistência técnica), Custo (gastos gerais de fabricação, desempenho do departamento através de uma relação entre produção e mão de obra), Atendimento (parada de linha; falta de peças; performance de atendimento da fábrica às solicitações da assistência técnica), Gerenciamento (absenteísmo; hora extra, evolução do número de pessoas ao longo do tempo) e Segurança (número de acidentes).

\subsection{Métodos para Empresas com Manufatura Enxuta já Implantada ou Não}

\subsubsection{Métodos que apresentam o instrumento de coleta de dados}

Dias, Fernandes e Godinho Filho (2004) apresentam uma metodologia baseada em indicadores de desempenho, para a avaliação da implantação da ME aplicada em uma indústria do setor médico hospitalar. A metodologia é composta por 4 passos: 1) Identificar o objetivo: refere-se aos objetivos de desempenho que são prioritários com relação à implementação da $\mathrm{ME}$, visando a melhoria da qualidade, produtividade, ou ambos; 2) Definir os indicadores de desempenho: são definidos os indicadores a serem utilizados dependendo da abrangência dos princípios implantados ou a serem implantados (chão de fábrica, empresa ou cadeia de suprimentos) e do grau de implantação (princípios já implantados - dados reais; ou que serão implantados - dados simulados); 3) Calcular os valores dos indicadores: após serem definidos os indicadores a serem utilizados em função dos objetivos, abrangência e grau de implantação, devem ser calculados os valores 
destes indicadores; 4) Analisar os indicadores: os resultados são analisados para avaliar a implantação da ME e verificar se ela está realmente contribuindo (ou irá contribuir) para a melhoria da competitividade da empresa.

Fernandes, Godinho Filho e Dias (2005) avaliam a implantação da ME por meio de indicadores de desempenho baseado no método de Dias, Fernandes e Godinho Filho (2004). A diferença é que foi acrescentado um passo a mais na sua condução: Definir a abrangência e o grau de implantação dos princípios da ME. Este passo, basicamente, já constava no método de Dias, Fernandes e Godinho Filho (2004), porém não estava explícito em seu passo a passo.

O método de Tubino et al. (2008), baseado na tese de Andrade (2006), identificado como Benchmarking Enxuto (BME), é utilizado para diagnosticar o estado atual do sistema produtivo, podendo também ser utilizado por empresas que ainda não tenham implantado a $M E$ e precisam de uma ferramenta de auxílio à implantação. A aplicação BME se dispõe a fornecer um referencial sobre como estão implantadas as práticas da ME em torno de quatro variáveis (demanda, produto, planejamento e controle da produção (PCP) e chão de fábrica) e verificar quais os desempenhos obtidos pelo sistema produtivo resultantes do nível de implantação destas práticas, tendo, como padrão de comparação (benchmark), os valores esperados para um sistema produtivo enxuto. $O$ método possui três etapas: etapa inicial de preparação, na qual se monta um grupo de pessoas e se criam as condições básicas para iniciar o trabalho; etapa de investigação, na qual são medidos os 37 indicadores que compõem o questionário utilizado como instrumento de coleta de dados; e etapa de interpretação na qual há o tratamento dos dados e discussão dos resultados alcançados, que são sustentados e analisados com uso de gráficos. O método de diagnóstico elaborado foi testado e validado, aplicando-se em cinco empresas de médio e grande porte no Estado de Santa Catarina.

Furini e Saurin (2008) apresentam um método para avaliar a cultura Lean da empresa por meio de um questionário com 15 questões baseadas nos 14 princípios de gestão do modelo Toyota (LIKER, 2005). É aplicado com gestores, lideranças e operadores de uma indústria de arames. A intenção do método é avaliar o quanto as pessoas estão capacitadas e conscientizadas sobre os conceitos e objetivos da implantação de práticas da ME na empresa. 
Tanto Dal Forno et al. (2009) quanto Silva, Elias e Tubino (2009) e Silva et al. (2010) utilizam o método proposto por Tubino et al. (2008). Dal Forno et al. (2009) aplicam o método em uma indústria do setor cerâmico mostrando seu posicionamento, com a determinação dos seus pontos fortes e fracos. Tais pontos são identificados a partir da análise das práticas e desempenho das variáveis do método para guiar um plano de ação para a implementação da ME na empresa. Já Silva, Elias e Tubino (2009) aplicam o método no processo de tecelagem de uma empresa têxtil de grande porte, que havia implantado a ME e utilizou a ferramenta para gerar um diagnóstico de seu estado atual. Silva et al. (2010) aplicam o método a uma indústria de aditivos químicos e impermeabilizantes, verificando o quão enxuta está a empresa e como se coloca em comparação com outras indústrias consideradas como referências.

Souza e Brandstetter (2010) analisam as práticas enxutas adotadas em 8 empresas de construção civil na região de Goiânia. Como instrumento de coleta de dados é utilizada uma lista de verificação em que são explorados quatro princípios definidos pelos autores como: 1) o aumento da transparência do processo, 2) a redução da variabilidade, 3) aumento da flexibilidade de saída e, 4) o valor do produto/serviço a partir das considerações dos clientes.

\subsubsection{Métodos que não apresentam o instrumento de coleta de dados}

Ferreira e Saurin (2005) propõem a versão preliminar de um método para avaliar de forma mais aprofundada o nível de implementação dos princípios da ME que foi aplicada em uma fábrica de máquinas agrícolas. Para a avaliação do nível de implementação das práticas da $\mathrm{ME}$, foi desenvolvida uma lista de verificação contendo 81 perguntas, distribuídas ao longo dos 14 princípios da produção enxuta, definidos pelos autores com base na literatura. Cada item da lista de verificação possui uma classificação quanto a sua aplicação: não se aplica (NA); não existe $(\mathrm{NE})$, correspondendo a itens que não estão sendo aplicados, mas que, devido às características da empresa, poderiam ser adotados; aplicação muito fraca (MFR); aplicação fraca (FR); aplicação forte (FO); e aplicação muito forte (MFO). Cada possibilidade de resposta da lista de verificação ainda possui um peso diferenciado que compõe a equação que calcula a nota de cada prática: $\mathrm{NE}=0,0 ; \mathrm{MFR}=2,5 ; \mathrm{FR}$ 
$=5,0 ; \mathrm{FO}=7,5 ; \mathrm{MFO}=10,0$. A nota alcançada por cada prática da lista de verificação é definida pela Equação 3:

$$
\text { Nota }=\underline{(B \times 2,5)+(C \times 5,0)+(D \times 7,5)+(E \times 10,0)}
$$

\section{A}

Onde: (A) é igual ao número de itens aplicáveis; (B) é igual ao número de itens com aplicação muito fraca; (C) é igual ao número de itens com aplicação fraca; (D) é igual ao número de itens com aplicação forte; e (E) igual ao número de itens com aplicação muito forte.

Silva, Araújo e Gomes (2009) utilizam o método de Ferreira e Saurin (2005) para avaliação das práticas da ME em uma indústria farmacêutica por meio de um estudo de caso.

Francisco e Hatakeyama $(2008,2009)$ propõem uma avaliação de dez práticas da ME, caracterizadas como ferramentas da ME. Na publicação de 2008, o método estava em fase de aplicação e os resultados não haviam sido tabulados. A publicação de 2009 apresenta o resultado do método que foi aplicado em 12 empresas do setor madeireiro, utilizando como instrumento de coleta de dados um questionário com 22 questões, embasadas nas dez ferramentas da $\mathrm{ME}$, caracterizadas pelos autores.

Dal Forno et al. (2008) utilizam especificamente a parte do método BME, proposto por Tubino et al. (2008). Tratam a variável produto como forma de investigar o grau de desenvolvimento do sistema produtivo, em relação às práticas e desempenho alcançados no processo de projeto do produto em uma indústria montadora de placas de circuito impresso.

\subsection{Métodos para Empresas com Manufatura Enxuta não implantada}

Quanto aos métodos para empresas com ME não implantada, foram identificados somente métodos que não apresentaram o instrumento de coleta de dados, descritos a seguir.

Sellitto, Borchard e Pereira (2003) descrevem o desenvolvimento e a aplicação de um indicador que permita avaliar o grau de aptidão de uma empresa ou uma indústria para receber uma abordagem estruturada capaz de conduzi-la a implementação dos princípios da ME. A metodologia proposta foi aplicada a uma 
indústria de serviços - 15 empresas de transporte coletivo, de Porto Alegre. O método é baseado em cinco constructos: (1) capacidade de compreender o que agrega valor ao cliente ou usuário do serviço; (2) capacidade de eliminar perdas na cadeia de produção de valor da indústria; (3) capacidade de tornar a operação previsível; (4) capacidade de permitir que o cliente "puxe" a operação; (5) capacidade de medir e melhorar o resultado continuamente. Associaram-se a cada construto seis variáveis que são medidas por questionários baseados na escala de Likert de 5 pontos, compreendendo de "totalmente negativo" a "totalmente positivo".

Sellitto et al. (2004), com base em Sellitto, Borchard e Pereira (2003), propõem construir um sistema de medição de desempenho capaz de ser o elo de realimentação de ações originadas em uma estratégia enxuta na busca de objetivos definidos no planejamento da indústria de transporte coletivo.

\section{ANÁLISE DAS PUBLICAÇÕES IDENTIFICADAS SOBRE AVALIAÇÃO DA MANUFATURA ENXUTA}

As análises realizadas nesta seção estão baseadas na classificação proposta na seção 3 e nos dados resultantes das Tabelas 1 e 2, que correspondem às 28 publicações identificadas no ENEGEP sobre avaliação da ME.

Quanto às práticas da ME enfatizadas pelos métodos, foi elaborada a Tabela 3 que apresenta a frequência de utilização destas práticas ordenadas em ordem decrescente.

Verifica-se através da Tabela 3 que as práticas enxutas mais abordadas nos métodos que avaliam a ME são Ferramentas da Qualidade e Equipes Multifuncionais, que aparecem em $75 \%$ dos métodos. Seguidas de Troca Rápida de Ferramentas (TRF) e Melhoria Contínua/Kaizen (62,5\% cada) e Gestão Visual/ Comunicação $(56,3 \%)$. 
Tabela 3 - Frequência de abordagem das práticas da Manufatura Enxtua dos métodos analisados

\begin{tabular}{lcc}
\hline \multicolumn{1}{c}{ Práticas da Manufatura Enxuta } & Frequência & $\begin{array}{c}\text { \% em Relação } \\
\text { ao Total de } \\
\text { Métodos }\end{array}$ \\
\hline Ferramentas da Qualidade & 12 & 75,0 \\
Equipes Multifuncionais & 12 & 75,0 \\
Troca Rápida de Ferramentas & 10 & 62,5 \\
Melhoria Contínua, Kaizen & 10 & 62,5 \\
Gestão Visual, Comunicação & 9 & 56,3 \\
Kanban, Sistema Puxado, JIT & 8 & 50,0 \\
5S & 8 & 50,0 \\
Operaçães Padronizadas & 8 & 50,0 \\
Manutenção Produtiva Total & 8 & 50,0 \\
Nivelamento da Produção & 8 & 50,0 \\
Agregação de Valor & 7 & 43,7 \\
Satisfação no Trabalho & 7 & 43,7 \\
Autonomação, Jidoka & 6 & 37,5 \\
Projeto/Desenvolvimento de produto flexível & 6 & 37,5 \\
Poka yoke & 5 & 31,3 \\
Fluxo Contínuo & 5 & 31,3 \\
Integração de Fornecedores & 5 & 31,3 \\
Andon & 4 & 25,0 \\
Segurança no Trabalho & 4 & 25,0 \\
Mapeamento do Fluxo de Valor & 3 & 18,8 \\
Layout de Fábrica & 3 & 18,8 \\
Tecnologia de Grupo & 2 & 12,5 \\
\hline Fonte: Os autores
\end{tabular}

Outras práticas utilizadas, que estão presentes na metade dos métodos, são Kanban/Sistema Puxado/JIT, 5S, Operações Padronizadas, Manutenção Produtiva Total e Nivelamento da Produção. Já algumas práticas são abordadas em um número menor de métodos, como no caso de Andon e Segurança no Trabalho (25,0\% cada), Mapeamento do Fluxo de Valor e Layout de Fábrica (18,8\% cada). A prática enxuta menos utilizada é Tecnologia de Grupo, com 12,5\%.

Quanto à metodologia científica empregada, 53,6\% (15 artigos) possuem a abordagem metodológica estudo de caso, 25\% (7 artigos) não mencionam o tipo de abordagem metodológica empregada, $17,8 \%$ são pesquisas do tipo survey (5 artigos) e 3,6\% (1 artigo) correspondem à pesquisa teórico-conceitual. Não foram encontrados trabalhos relacionados às metodologias experimentos, modelagem, simulação e pesquisa-ação. Estes resultados corroboram com Berto e Nakano (2000) e Miguel (2007), os quais afirmam que o estudo de caso é a abordagem metodológica mais utilizada na área de Engenharia de Produção no Brasil.

A ausência de experimentos, modelagem e simulação provavelmente se deve ao fato de que todos os estudos realizados propuseram um método para ser conduzidos na prática em organizações reais. A ausência da abordagem pesquisa- 
ação deve-se ao fato de que, considerando todos os autores de cada trabalho, havia, pelo menos, um que possuía vínculo direto com a universidade. Desta forma, não pode ser caracterizado como pesquisa-ação um método que conta com o desempenho ativo dos pesquisadores para atuar na resolução dos problemas encontrados, gerando ações e propostas que são expostas a todo grupo participante interessado, além de ser conduzida com a presença de todos os pesquisadores em tempo real (BARROS; LEHFELD, 2000; COUGHLAN; COGHLAN, 2002).

Dos métodos que foram aplicados na prática (25 dos 28 artigos), a presença do pesquisador foi constatada em mais da metade dos estudos analisados (56\%) e sua ausência foi caracterizada em 5 estudos (20\%). Em 6 artigos, os autores não deixaram explícitos se o método foi aplicado ou não, e se contou com a presença do pesquisador.

Apenas 12 publicações (menos da metade, 42,8\%) apresentaram 0 instrumento de coleta de dados completo, o que significa que seria impossível aplicar o método de avaliação da ME com a simples leitura do artigo. Assim, o método não seria útil, por exemplo, para empresas que estivessem interessadas em aplicá-lo sem contar com o auxílio dos pesquisadores que o desenvolveram.

Quanto às formas de apresentação de resultados gerada pelos métodos analisados, apenas 4 (PANTALEÃO; ANTUNES JÚNIOR, 2003; SATOLO; CALARGE, 2007; TUBINO et al., 2008; ROSA et al., 2010a) apresentam alguma classificação ou grau de escala de implementação da ME após sua aplicação (se forem consideradas todas as publicações, este número passa para 8, visto que alguns métodos são utilizados em mais de uma publicação). Nas demais pesquisas, os métodos fazem análises descritivas e relatos das situações encontradas na avaliação.

Além das considerações feitas, baseadas nas Tabelas 1, 2 e 3, cabe destacar também:

a) Apenas 4 publicações não foram aplicadas na prática (PANTALEÃO; ANTUNES JÚNIOR, 2003; FRANCISCO; HATAKEYAMA, 2008; FURINI; SAURIN, 2008; NOGUEIRA; CASALINHO, 2008) e apenas 2 apresentam um modelo quantitativo de análise da ME (FERREIRA; SAURIN, 2005; SATOLO; CALARGE, 2007); 
b) Das 28 publicações apresentadas, apenas 4 (GIESTA; MAÇADA, 2002; GIESTA, MAÇADA; LUNARD, 2003; BATISTA; MUNIZ; BATISTA JÚNIOR, 2008; FURINI; SAURIN, 2008) estão focadas em aspectos humanos, ou seja, relacionam aspectos à força de trabalho na avaliação da ME. Destaque também aos métodos de Giesta e Maçada (2002); Dias, Fernandes e Godinho Filho (2004); Satolo e Calarge (2007) e Tubino et al. (2008), que são encontrados em mais de uma publicação;

c) Outra importante consideração a fazer é que apenas 3 das 28 publicações analisaram um setor diferente da indústria (SELLITTO; BORCHARD; PEREIRA, 2003; SELLITTO et al., 2004; SOUZA; BRANDSTETTER, 2010), enquanto demais publicações estavam focadas na implantação da ME no chão de fábrica, o que revela ainda grande predominância no Brasil da adoção da ME nas áreas manufatureiras;

d) Quanto ao número de publicações por universidade, a Universidade Federal do Rio Grande do Sul e a Universidade Federal de Santa Catarina foram as que mais tiveram publicações ( 5 artigos cada uma), seguidas da Universidade Estadual Paulista e Universidade do Vale do Rio dos Sinos (3 artigos cada uma). As demais universidades que tiveram 2 artigos publicados foram: a Universidade Federal de São Carlos, Universidade Metodista de Piracicaba, Universidade Estadual de Ponta Grossa, Universidade Tecnológica Federal do Paraná e Universidade Federal do Rio Grande.

\section{CONCLUSÕES}

Por meio deste trabalho, foi possível identificar o perfil da pesquisa brasileira sobre avaliação da implantação da ME, baseando-se em publicações dos últimos dez anos de realização do ENEGEP. Nesta análise foram consideradas apenas publicações que avaliam a ME de forma mais abrangente, ou seja, não foram incluídas publicações que relatavam os resultados de aplicações isoladas, tais como a implantação de Kanban ou a utilização de Troca Rápida de Ferramentas. 
Esta pesquisa resultou em 28 artigos que correspondem a 16 métodos de avaliação da implantação da $\mathrm{ME}$, pois alguns métodos foram encontrados em mais de um artigo.

A partir das características dos artigos identificados na revisão dos Anais do ENEGEP, foi possível sugerir um sistema de classificação baseado em seis categorias. São elas: práticas da $\mathrm{ME}$, abordagem metodológica, método para $\mathrm{ME}$ implantada, presença do pesquisador na aplicação do método, apresentação do instrumento de coleta de dados e apresentação de alguma classificação ou grau de escala de implementação da ME na empresa avaliada.

Esse procedimento serviu como base para a estruturação de toda a revisão bibliográfica e para a análise dos artigos que abordam métodos de avaliação da ME.

O número de publicações que avaliam a implantação da $\mathrm{ME}$, se comparado com o de publicações que relatam sua implantação, pode significar uma lacuna a ser explorada, no que diz respeito à elaboração e desenvolvimento de novos métodos de avaliação.

A mesma suposição é válida para a quantidade de métodos que abordam uma classificação de quão enxuta se apresenta a empresa avaliada. Este tipo de diagnóstico pode auxiliar as empresas a avaliarem o progresso de seus esforços em direção à ME.

A principal contribuição deste trabalho, além de mostrar o perfil da pesquisa de avaliação da ME no Brasil, é gerar uma perspectiva de como devem ser conduzidos os próximos trabalhos nesse mesmo tema, para que evoluam a partir das características e aspectos importantes dos métodos já existentes.

\section{REFERÊNCIAS}

AHLSTRÖM, P. Sequences in the implementation of lean production. European Management Journal, v.16, n.3, p. 327-334, 1998.

ANDRADE, G. J. P. O. Um método de diagnóstico do potencial de aplicação da Manufatura Enxuta na indústria têxtil. 2006. 297f.Tese (Doutorado em Engenharia de Produção) - Programa de Pós-Graduação em Engenharia de Produção, Universidade Federal de Santa Catarina, Florianópolis, 2006.

\section{BARROS, A. J. S.; LEHFELD, N. A. S. Fundamentos da metodologia científica.} 2.ed. São Paulo: Makron Books, 2000. 
BATISTA, J. B.; MUNIZ, J.; BATISTA JÚNIOR, E. D. Análise do Sistema Toyota de Produção: estudo exploratório em empresas brasileiras do grupo Toyota. In: ENCONTRO NACIONAL DE ENGENHARIA DE PRODUÇÃOO, 28., 2008. Rio de Janeiro. Anais... Rio de Janeiro, 2008.

BERTO, R. M. V. S.; NAKANO, D. N. A produção científica nos anais do encontro nacional de engenharia de produção: um levantamento de métodos e tipos de pesquisa. Produção, v. 9, n. 2, p. 65-76, 2000.

BORGES JÚNIOR, C. A. ; BARROS, J. G. M.; REIS, A. C. C. PALMEIRA, A. A. Avaliação da melhoria de performance decorrente da implantação da manufatura enxuta na planta s-10 da General Motors do Brasil. In: ENCONTRO NACIONAL DE ENGENHARIA DE PRODUÇÃO, 24, 2004. Florianópolis. Anais... Florianópolis, 2004.

CALARGE, F. C.; SATOLO, E. G.; PEREIRA, F. H. Avaliação de implementação do Lean Production baseada na norma SAE J4000: uma análise em empresas do setor automotivo de Brasil e Espanha. In: ENCONTRO NACIONAL DE ENGENHARIA DE PRODUÇÃO, 29, 2009. Salvador. Anais... Salvador, 2009.

CARDOZA, E.; CARPINETTI, L. C. R. Indicadores de desempenho para o sistema de produção enxuto. Produção Online. v. 5, n. 2, 2005. Disponível em: $<$ http://producaoonline.org.br/index.php/rpo/article/view/338/433>. Acesso em: 20 dez. 2010.

CEZAR, G. M.; MOITA, M. H. V. Avaliação da eficácia da aplicação das ferramentas e filosofia Lean em uma multinacional japonesa de grande porte em processo de expansão contínua. In: ENCONTRO NACIONAL DE ENGENHARIA DE PRODUÇÃO, 29, 2009. Salvador. Anais... Salvador, 2009.

COUGHLAN, P.; COGHLAN, D. Action Research for Operation Management. International Journal of Operations \& Production Management, v. 22, n. 2, p. 220-240, 2002.

DAL FORNO, A. J.; BUSON, M. A.; SCHUCH, C. G.; FORCELLINI, F. A.; FERREIRA, M. G. G. O processo de desenvolvimento de produtos sob a ótica Lean: a variável produto do Benchmarking Enxuto. In: ENCONTRO NACIONAL DE ENGENHARIA DE PRODUÇÃO, 28, 2008. Rio de Janeiro. Anais... Rio de Janeiro, 2008.

DAL FORNO, A. J.; TUBINO, D. F.; FORCELLINI, F. A.; PEREIRA, F. A. Aplicação da ferramenta Benchmarking Enxuto: estudo de caso em uma empresa do setor cerâmico. In: ENCONTRO NACIONAL DE ENGENHARIA DE PRODUÇÃO, 29, 2009. Salvador. Anais... Salvador, 2009.

DIAS, F. T.; FERNANDES, F. C. F; GODINHO FILHO, M.; Uma metodologia baseada em indicadores de desempenho para avaliação de princípios relativos à Manufatura Enxuta: estudo de caso em uma empresa do setor médico-hospitalar. In: 
ENCONTRO NACIONAL DE ENGENHARIA DE PRODUÇÃO, 24, 2004, Florianópolis. Anais... Florianópolis, 2004.

DURAN, O.; BATOCCHIO, A. Na direção da manufatura enxuta através da J4000 e o LEM. Produção Online, v. 3, n. 2, 2003. Disponível em: <

http://producaoonline.org.br/index.php/rpo/article/view/619/657>. Acesso em: 20 dez. 2010.

FERNANDES, F. C. F.; GODINHO FILHO, M.; DIAS, F. T. Proposta de um método baseado em indicadores de desempenho para avaliação dos princípios relativos a Manufatura Enxuta. In: ENCONTRO NACIONAL DE ENGENHARIA DE PRODUÇÃO, 25, 2005. Porto Alegre. Anais... Porto Alegre, 2005.

FERREIRA, C. F.; SAURIN, T. A. Avaliação do nível de implementação de capacitadores da produção enxuta em uma linha de montagem de colheitadeiras. In: ENCONTRO NACIONAL DE ENGENHARIA DE PRODUÇÃO, 25, 2005. Porto Alegre. Anais... Porto Alegre, 2005.

FILIPPINI, R. Operations management research: some reflections on evolution, models and empirical studies in OM. International Journal of Operations \& Production Management, v. 17, n. 7, p. 655-670, 1997.

FRANCISCO, B. R.; HATAKEYAMA, K. Diagnóstico sobre a aplicação do método de produção enxuta no ramo madeireiro. In: ENCONTRO NACIONAL DE ENGENHARIA DE PRODUÇÃO, 28, 2008. Rio de Janeiro. Anais... Rio de Janeiro, 2008.

FRANCISCO, B. R.; HATAKEYAMA, K. Diagnóstico da utilização de 10 ferramentas da produção enxuta no ramo madeireiro. In: ENCONTRO NACIONAL DE ENGENHARIA DE PRODUÇÃO, 29., 2009. Salvador. Anais... Salvador, 2009.

FURINI, G.; SAURIN, T. A. Proposta de um método de análise da cultura Lean em uma empresa que está implantando práticas do sistema de produção enxuta. In: ENCONTRO NACIONAL DE ENGENHARIA DE PRODUÇÃO, 28, 2008. Rio de Janeiro. Anais... Rio de Janeiro, 2008.

GIESTA, L. C.; MAÇADA, A. C. G.; LUNARDI, G. L. Sistema de produção enxuta: análise em empresas do setor metalmecânico do Rio Grande do Sul. In:

ENCONTRO NACIONAL DE ENGENHARIA DE PRODUÇÃO, 23, 2003. Ouro Preto. Anais... Ouro Preto, 2003.

GIESTA, L. C.; MAÇADA, A. C. G. Validação de um instrumento para analisar o sistema de produção enxuta (SPE) na percepção dos funcionários. In: ENCONTRO NACIONAL DE ENGENHARIA DE PRODUÇÃO, 22, 2002. Curitiba. Anais... Curitiba, 2002.

GIL, A. C. Como elaborar projetos de pesquisa. 4.ed. São Paulo: Atlas, 2010. 
IMAI, M. Kaizen: a estratégia para o sucesso competitivo. 5.ed. São Paulo: IMAM, 1994.

KARLSSON, C.; AHLSTRÖM, P. Assessing changes towards lean production. International Journal of Operations \& Production Management, v. 16, n. 2, p. 2141, 1996.

LIKER, J. K. O Modelo Toyota: 14 princípios de gestão do maior fabricante do mundo. São Paulo: Bookman, 2005.

LUCATO, W. C.; MAESTRELLI, N. C.; VIEIRA JÚNIOR, M. Determinação do grau de enxugamento de uma empresa: uma proposta conceitual. Ciência \& Tecnologia, v. 12, n. 24, p. 25-38, 2006.

MORIONES, A. B.; PINTADO, A. B.; CERIO, J. M. D. The role of organizational context and infrastructure practices in JIT implementation. International Journal of Operations \& Production Management, v. 28, n. 11, p.1042-1066, 2008.

MARODIN, G.; SAURIN, T. A. Diretrizes para gestão de barreiras na implantação de sistemas de produção enxuta. In: ENCONTRO NACIONAL DE ENGENHARIA DE PRODUÇÃO, 30, 2010, São Carlos. Anais... São Carlos, 2010.

MEDEIROS, J. B. Redação científica. 9.ed. São Paulo: Atlas, 2007.

MIGUEL, P. A. C. Estudo de caso na engenharia de produção: estruturação e recomendações para sua condução. Produção. v. 17, n. 1, p. 216-229, 2007.

NOGUEIRA, M. G. S.; CASALINHO, G. D. A. O. Proposta de uma matriz de indicadores para avaliação de desempenho de práticas enxutas. In: ENCONTRO NACIONAL DE ENGENHARIA DE PRODUÇÃO, 28, 2008. Rio de Janeiro. Anais... Rio de Janeiro, 2008.

NOGUEIRA, M. G. S.; SAURIN, T. A. Avaliação do nível de implementação de práticas enxutas com base nas percepções dos gerentes. In: ENCONTRO NACIONAL DE ENGENHARIA DE PRODUÇÃO, 26, 2006. Fortaleza. Anais... Fortaleza, 2006.

OHNO, T. O sistema Toyota de produção. Além da produção em larga escala. Porto Alegre: Artes Médicas, 1997.

PANTALEÃO, L. H.; ANTUNES JÚNIOR, J. A. V. Avaliação da aprendizagem organizacional a respeito do Sistema Toyota de Produção/Lean Production System: uma proposição metodológica. In: ENCONTRO NACIONAL DE ENGENHARIA DE PRODUÇÃO, 23, 2003, Ouro Preto. Anais... Ouro Preto, 2003.

ROSA, A. B.; SOUZA, F. B.; SALGADO, M. H.; ALVES, T. Q. Estudo sobre relação entre a adoção das práticas de manufatura enxuta e a adoção de prioridades competitivas em empresas de manufatura: uma pesquisa survey nas empresas da 
região de Bauru-SP. In: ENCONTRO NACIONAL DE ENGENHARIA DE PRODUÇÃO, 30, 2010, São Carlos. Anais... São Carlos, 2010a.

ROSA, A. B.; SOUZA, F. B.; SALGADO, M. H.; ALVES, T. Q. Estudo exploratório sobre nível de adoção de práticas da manufatura enxuta na região de Bauru-SP. In: ENCONTRO NACIONAL DE ENGENHARIA DE PRODUÇÃO, 30, 2010. São Carlos. Anais... São Carlos, 2010b.

ROTHER, M.; HARRIS, H. Criando fluxo contínuo: um guia de ação para gerentes, engenheiros e associados da produção. São Paulo: Lean Institute Brasil, 2002.

SÁNCHEZ, M. A.; PÉREZ, M. P. Lean indicators and manufacturing strategies. International Journal of Operations \& Production Management, v. 21, n. 11, p.1433-1451, 2001.

SATOLO, E. G.; CALARGE, F. A. Determinação do grau de aderência ao sistema lean production para empresas da indústria automobilística: um estudo tipo. In: ENCONTRO NACIONAL DE ENGENHARIA DE PRODUÇÃO, 27, 2007. Foz do Iguaçu. Anais... Foz do Iguaçu, 2007.

SAURIN, T. A.; FERREIRA, C. F. Avaliação qualitativa da implantação de práticas da produção enxuta: estudo de caso em uma fábrica de máquinas agrícolas. Gestão \& Produção, v. 15, n. 3, p. 449-462, 2008.

SAURIN, T. A.; FERREIRA, C. F. The impacts of lean production on working conditions: a case study of a harvester assembly line in Brazil. International Journal of Industrial Ergonomics, v. 39, n. 2, p. 403-412, 2009.

SELLITTO, M.; BORCHARD, M; PEREIRA, G. M. Avaliação do grau de preparação de uma indústria para receber uma abordagem que a leve em direção à Mentalidade Enxuta. In: ENCONTRO NACIONAL DE ENGENHARIA DE PRODUÇÃO, 23, 2003, Ouro Preto. Anais... Ouro Preto, 2003.

SELLITTO, M.; BORCHARD, M; PEREIRA, G. M.; OLIVEIRA, G. Graus de importância de critérios de desempenho de uma estratégia enxuta aplicada em uma indústria de serviços. In: ENCONTRO NACIONAL DE ENGENHARIA DE PRODUÇÃO, 24, 2004. Florianópolis. Anais... Florianópolis, 2004.

SHINGO, S. O Sistema Toyota de produção: do ponto de vista da engenharia de produção. Porto Alegre: Bookman, 1996.

SHINGO, S. Sistema de troca rápida de ferramenta. uma revolução nos sistemas produtivos. Porto Alegre: Bookman, 2000.

SILVA, A. M.; ARAÚJO, A. C.; GOMES, M. L. B. Avaliação das práticas de produção enxuta numa empresa de produtos farmacêuticos. In: ENCONTRO NACIONAL DE ENGENHARIA DE PRODUÇÃO, 29, 2009. Salvador. Anais... Salvador, 2009. 
SILVA, G. S.; ELIAS, S, J. B; TUBINO, D. F. Diagnóstico do estágio Lean de uma indústria têxtil de grande porte. In: ENCONTRO NACIONAL DE ENGENHARIA DE PRODUÇÃO, 29., 2009. Salvador. Anais... Salvador, 2009.

SILVA, L.; NEVES, C. A. A.; TUBINO, D. F.; MERINO, E. A. D.; SELIG, P. M. Aplicação do método Benchmarking Enxuto em uma indústria de aditivos químicos e impermeabilizantes do segmento da construção civil. In: ENCONTRO NACIONAL DE ENGENHARIA DE PRODUÇÃO, 30, 2010. São Carlos. Anais... São Carlos, 2010.

SOUZA, S. L.; BRANDSTETTER, M. C. G. O. Avaliação de princípios da Lean Construction em construtoras Goianas. In: ENCONTRO NACIONAL DE ENGENHARIA DE PRODUÇÃO, 30, 2010. São Carlos. Anais... São Carlos, 2010.

SORIANO-MEIER, H.; FORRESTER, P. L. A model for evaluating the degree of leanness of manufacturing firms. Integrated Manufacturing Systems, v. 13, n. 2, p. 104-109, 2002.

TAKAHASHI, Y.; OSADA, T. TPM/MPT. Manutenção produtiva total. São Paulo: IMAM, 1993.

TUBINO, D. F.; SILVA, G. G. M. P.; ANDRADE, G. J. P. O.; HORNBURG, S; OLIVEIRA, L. M. Benchmarking Enxuto: um método de auxílio à implantação da manufatura enxuta. In: ENCONTRO NACIONAL DE ENGENHARIA DE PRODUÇÃO, 28, 2008. Rio de Janeiro. Anais... Rio de Janeiro, 2008.

WOMACK, J. P.; JONES, D. T.; ROOS, D. A Máquina que mudou o mundo. Rio de Janeiro: Campus, 1992.

WOMACK, J. P.; JONES, D. T. A Mentalidade enxuta nas empresas. Rio de Janeiro: Campus, 1992.

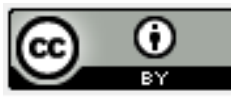

Artigo recebido em 23/12/2010 e aceito para publicação em 19/06/2012. 